\title{
Eichhornia crassipes (Mart) Solms en el Parque Nacional Marítimo-Terrestre das Illas Atlánticas de Galicia como resultado de un transporte por mar a larga distancia
}

Recibido: 23 xullo 2014 / Aceptado: 11 febreiro 2015

(C) IBADER- Universidade de Santiago de Compostela 2014

\begin{abstract}
Resumen Se analiza la aparición del jacinto de agua (Eichhornia crassipes (Mart) Solms) en las playas del archipiélago de las Islas Cíes en el Parque Nacional Marítimo-Terrestre das Illas Atlánticas de Galicia, como resultado de un posible transporte a larga distancia procedente de poblaciones naturalizadas situadas en el litoral de Portugal. La especie, nativa de América del Sur, presenta una gran capacidad invasora, vinculada a su gran capacidad reproductiva y de crecimiento. Está incluida en el listado de IUCN de las 100 especies invasoras más dañinas del planeta. El Jacinto de Agua parece citado por primera vez en Europa en la zona centro de Portugal, durante la primera mitad del siglo $X X$, en donde se ha ido expandiendo principalmente por las cuencas de los ríos Tejo y Sado, aunque también se conocen poblaciones en la zona norte. En Galicia su presencia es testimonial y las escasas citas acreditadas, aparecen asociadas a la posterior erradicación de los ejemplares. Entre los años 2011 y 2012 y tras el paso de fuertes borrascas procedentes del suroeste, se detectó la presencia en una de las playas del archipiélago de las Islas Cíes de restos de Eichhornia crassipes, que en base a la gran cantidad de los mismos y su estado de conservación, hacen pensar en un transporte de larga distancia desde una población de gran tamaño.
\end{abstract}

Pablo Ramil Rego · Marco Rubinos Román · Luis Gómez-Orellana - Boris Hinojo Sánchez

GI-1934 Territorio-Biodiversidade. IBADER - Universidade de

Santiago. Lugo

E-mail: ibader-ttb@gmail.com

\section{Patricia Rodríguez}

Reserarh.- managing biodiversity in forest, aquatic and agroecosystems (Centro de Estudos Florestais). Instituto Superior de Agronomía. Universidade Técnica de Lisboa
Palabras clave Jacinto de agua, Eichhornia crassipes, Parque Nacional marítimo-terrestre de las Illas Atlánticas de Galicia, invasión, transporte larga distancia.

Abstract The arrival of Water Hyacinth plants (Eichhornia crassipes (Mart) Solms.) at the beaches of theGalician Atlantic Islands Maritime-Terrestrial National Park is assessed as a consequence, of a long distance transport of individuals coming from naturalized populations located on the Portuguese coast. This South American native species is highly invasive due to its fast growing and high reproductive capacity. Water hyacinth is named by the IUCN as one of the top one hundred of the world's worst invasive alien species. It was first cited in Europe during the first half of the 20th century, in central Portugal, from where it has expanded mostly throughout the catchments of Tejo and Sado Rivers, although there are some other known populations in the north of Portugal. Its presence is quite exceptional in Galicia, being scarcely cited as it is normally linked to eradication events. During 2011 and 2012, after some SW squalls had passed by, some Eichhornia crassipes (Mart) Solms. remains were detected in one of the beaches of Cíes Island. Based upon the amount of plants, and the deterioration degree of the remains, it is thought that they have been transported from a big distant population.

Key words Water Hyacinth, Galician Atlantic Islands Maritime-Terrestrial National Park, invasion, long-distance transport.

\section{Introducción}

Las características biogeográficas del territorio costero atlántico de la Península Ibérica, determinan que las corrientes marinas y los vientos locales predominantes a lo largo de la costa del Norte de Portugal y de la zona atlántica de Galicia se dirijan hacia el norte. De este modo, se estima que cerca del $80 \%$ de los sedimentos en la plataforma 
continental atlántica de Galicia procedan del río Douro (Dias et al. 2002). La importancia de estas corrientes queda marcada en los propios sedimentos marinos, en cuyo interior se pueden encontrar microfósiles y restos orgánicos de especies procedentes tanto del Sur de la Península, como del Norte de África, y cuya individualización e interpretación genera importantes dificultades a nivel ecológico (Gómez-Orellana et al. 2013).

Este fenómeno es igualmente conocido por los marineros y pescadores, ya que estas corrientes determinan la distribución de los principales caladeros y las antiguas rutas empleadas por la navegación a vela. La magnitud de estas corrientes (10-15 Km/24 h), se ve incrementada en ocasiones de forma muy considerable por la concurrencia de condiciones climáticas particulares, como fuertes temporales.

La coincidencia de ambos factores, quedó patente en uno de los accidentes más trágicos que conmocionó en el año 2011 a Portugal y a toda Europa, derivado del colapso de un puente sobre el río Douro, cerca de la localidad de Castelo de Paiva, a unos $40 \mathrm{Km}$ de su desembocadura.

El hundimiento del puente, arrastró a tres turismos y un autobús. Parte de los cadáveres fueron transportadas por el río y alcanzaron el mar, donde las corrientes marinas los transportaron de forma rápida hacia el Norte, pudiendo ser recuperados en diversas localidades atlánticas de Galicia, así como distintos restos del autobús en el que viajaban. De este modo en 3-4 días recorrieron una distancia de más de $240 \mathrm{Km}$. Días más tarde, se recuperó en el mar un séptimo cadáver, en el litoral cantábrico de Galicia, en las proximidades de Estaca de Bares, a más de $410 \mathrm{Km}$ del accidente (Fig. 1).

En el trabajo se presentan los datos que permiten apoyar que estas mismas corrientes serían las responsables de la llegada a las playas de las Islas Cíes, en el Parque Nacional marítimo-terrestre de las Illas atlánticas de Galicia, de los restos verdes de una planta acuática, el jacinto de agua o camalote (Eichhornia crassipes).

\section{Antecedentes \\ Jacinto de Agua (Eichhornia crassipes (Mart) Solms)}

El jacinto de agua o camalote, designado por los aborígenes guaraníes como aguapé o aguapey es una planta acuática, monocotiledónea, perteneciente a la familia Pontederiaceae, originaria de América del Sur, en concreto, de las cuencas del río Amazonas y del río de la Plata.

Son plantas perennes con un tallo muy corto, de unos pocos centímetros, del que surgen grandes hojas dispuestas en roseta. Las hojas presentan largos peciolos y láminas circulares o más anchas que largas, de 2,5-16 cm de largo y $3-12 \mathrm{~cm}$ de ancho. En las hojas sumergidas, el peciolo es largo y cilíndrico, mientras que en las flotantes es más corto y se muestra muy hinchado, lo que facilita la flotabilidad de la planta (Fig. 2). De los nudos en la parte inferior del tallo surgen un conjunto de raíces de color marrón negruzco que llegan a alcanzar los $150 \mathrm{~cm}$ de longitud.

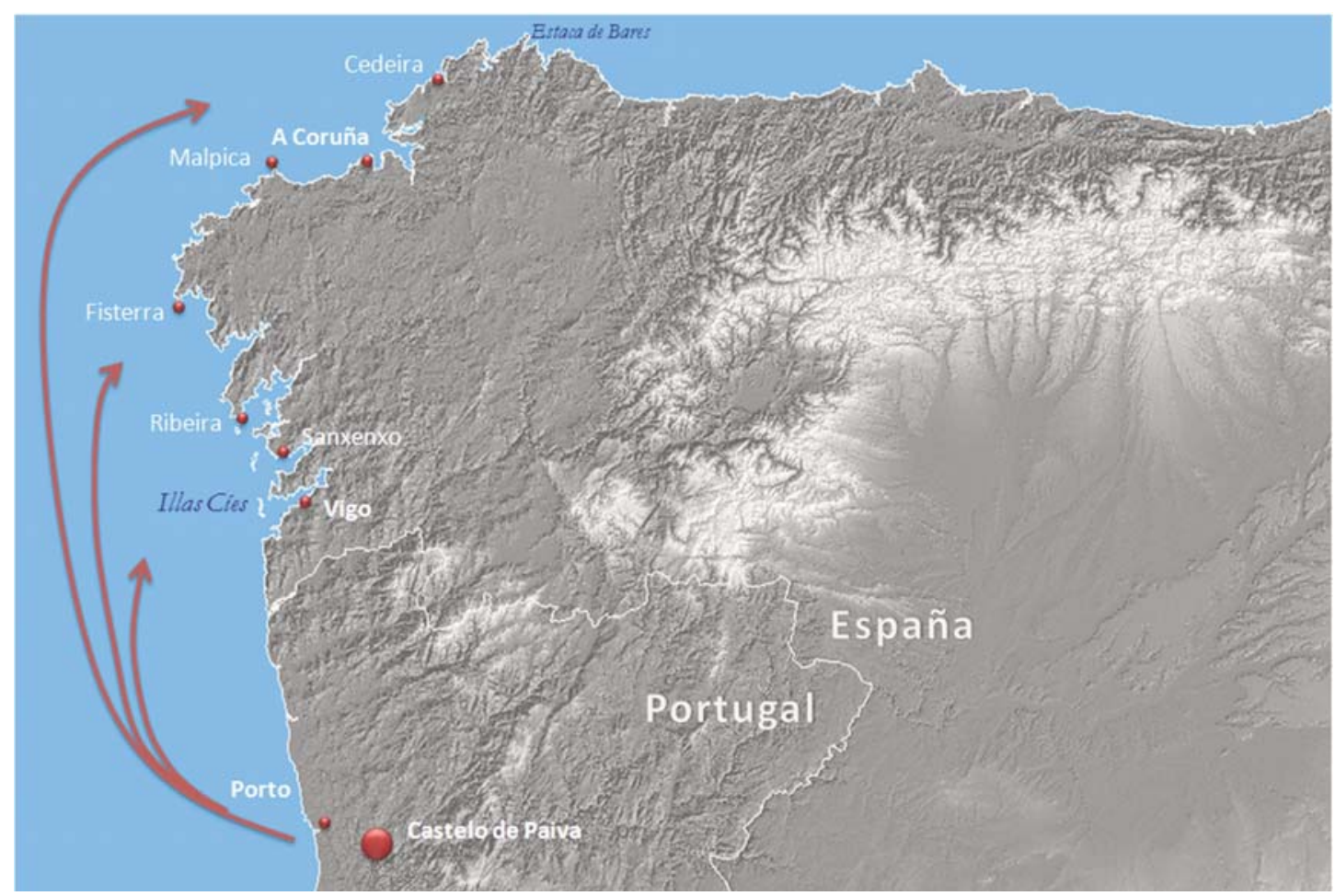

Figura 1.- Localización del territorio analizado y esquema del posible recorrido de los cuerpos de las víctimas del derrumbe del puente del río Douro hasta la costa de Galicia 


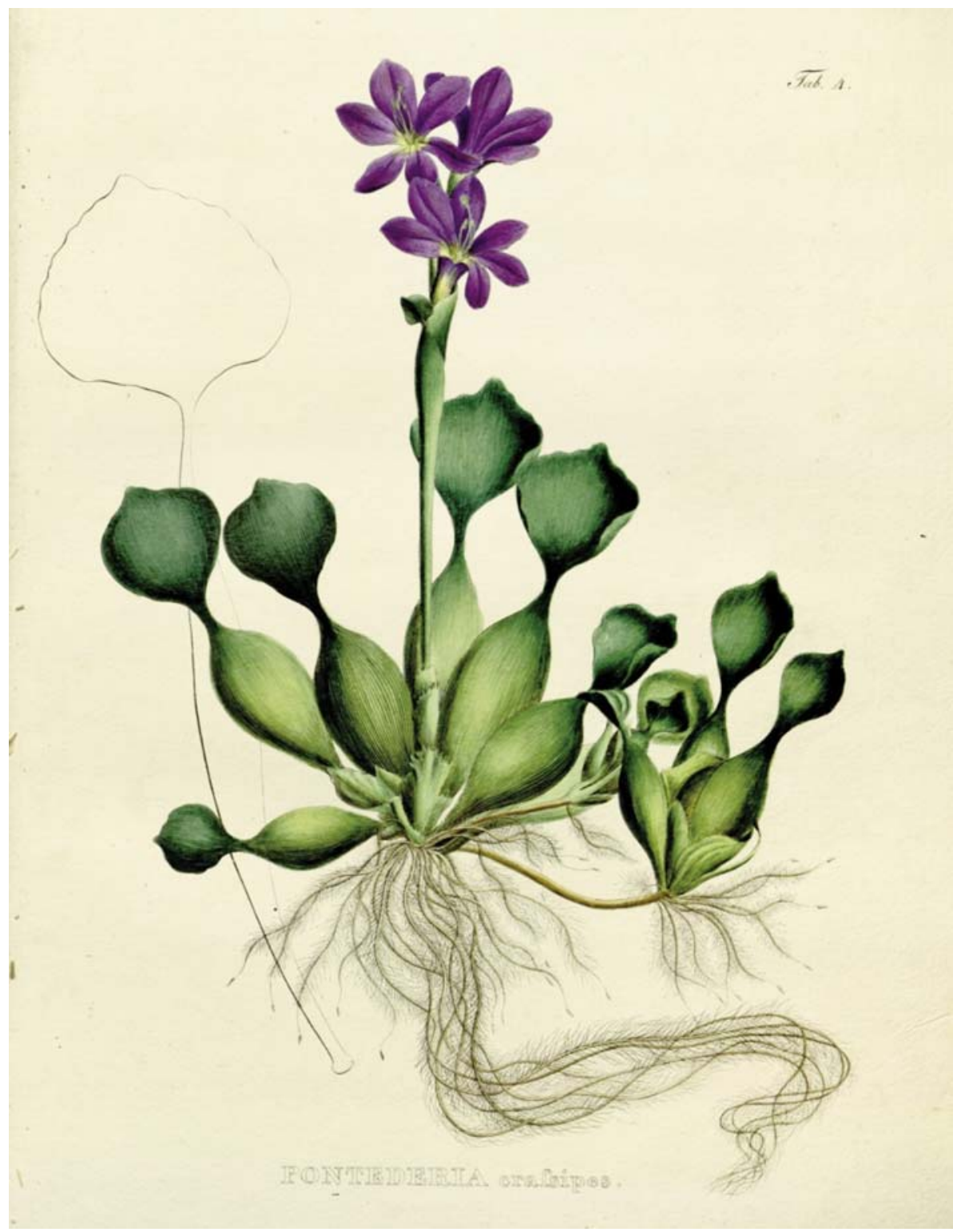

Figura 2.- Lámina de jacinto de agua (Eichhornia crassipes) publicada por von Martius en Nova Genera et Species Plantarum Brasiliensium (1823-1832)
Las flores de tonos azules a morados, raras veces blancas, alcanza los $5 \mathrm{~cm}$ y se asemejan en aspecto a la de los jacintos (Hyacinthus L.). Se encuentran agrupadas en espigas conformadas por 4-16 flores. Cada flor posee 6 piezas, 3 externas y 3 internas que protegen los 6 estambres y un único pistilo. El fruto es una cápsula elíptica, de más o menos $1,5 \mathrm{~cm}$ de largo, con 3 ángulos. Las semillas numerosas, de poco más de $1 \mathrm{~mm}$ de largo, con 10 costillas longitudinales.

Se reproduce activamente tanto por semilla como asexualmente (estolones, fragmentación de plantas). Las semillas, de las cuales se cita su posible dispersión por las aves, pueden conservar su capacidad germinativa entre 5 y 20 años (Sanz-Elorza et al. 2004).

El jacinto de agua habita principalmente en aguas estancadas (lagos, lagunas, estanques), aunque puede encontrarse en aguas corrientes en las que la velocidad de flujo del agua sea reducida (ríos, canales, zanjas de drenaje, etc.). Las condiciones para su desarrollo vienen marcadas fundamentalmente por aspectos climáticos al ser una especie muy sensible a los inviernos fríos y a las bajas temperaturas, su rango de desarrollo se establece en zonas con temperaturas medias entre $18-30{ }^{\circ} \mathrm{C}$, con un óptimo entre $22-25^{\circ} \mathrm{C}$, desapareciendo en áreas con importantes fríos invernales, con frecuentes heladas o en zonas donde se produce habitualmente la congelación de los medios acuáticos.

Los niveles de temperatura y nutrientes en el agua determinan el potencial peligro ambiental del Jacinto de Agua (Wilson et al., 2005). La falta de nutrientes ( $N$ y P) y períodos prolongados con temperaturas por debajo de los 5 ${ }^{\circ} \mathrm{C}$ o por encima de los $34{ }^{\circ} \mathrm{C}$, retrasan su crecimiento y pueden suponer sus límites de dispersión (Gopal, 1987; Owens \& Madsen, 1995; Sato, 1988). 
Otro de los factores que controlan su distribución es la calidad de agua, prefiriendo medios ricos en nutrientes (eutróficos o mesotróficos), de pH neutro o básico. Son especies intolerantes a las aguas marinas, aunque pueden colonizar estuarios con baja salinidad.

\section{Expansión territorial y efectos ambientales}

El descubrimiento científico del jacinto de agua fue realizado por el botánico alemán Carl Friedrich Philipp von Martius (17/04/1794-13/12/1868) y publicada en su trabajo Plantarum Brasiliensium Nova genera et species novae, vel minos cognitae (1823-1832).

Dada la vistosidad de sus flores, a finales del siglo XIX se inicia su expansión territorial, como planta ornamental, introduciéndose en jardines y estanques de América del Norte (USA en 1884) y del Sur, Japón e Indonesia. En 1890 es introducida en Australia. Su llegada a Europa y África es más reciente, en la primera mitad del siglo $X X$. En Europa únicamente se conocen poblaciones en Italia, España y Portugal. La cita más antigua corresponde con una población en la cuenca del río Sado, en Portugal, en el año 1940 (de Almeida \& Freitas, 2006). En el país luso la especie es considerada altamente infestante y tuvo una fuerte expansión a partir del año 1974, especialmente en el centro del país, en las cuencas hidrográficas del Tejo y Sado, y en menor medida en el norte y sur (San Elorza et al., 2004; Amaral Franco \& Rocha Alfonso, 1994)

A partir de un número muy limitado de introducciones, el jacinto de agua fue propagado y extendido por jardineros y particulares para ornamentar estanques y canales. En algunos territorios de Asia, África y América del Sur, su difusión se vio además favorecida al ser empleado como elemento para la obtención de biomasa destinada bien a la alimentación del ganado, producción de biogás o como abono (Ding et al. 2001; GIC, 2006). En otros casos su cultivo se relacionó con la capacidad filtradora de la planta, que debido a su rápido desarrollo es capaz de extraer del agua cantidades importantes de nutrientes y de ciertos contaminantes, o como alimento para el mantenimiento de otra especie invasora, la carpa.

Desde estos medios se expandió de forma natural a través de sus semillas, o pequeños fragmentos vegetativos, invadiendo acequias de regadío o drenaje, canales, tramos fluviales, lagos y charcas, desplazando a las comunidades nativas y transformando las características estructurales y funcionales de los ecosistemas acuáticos que invade.

La invasión territorial ha sido propiciada por un conjunto de factores vinculados tanto a la biología de la especies como a la acción humana. El jacinto de agua, es un planta que posee una fotosíntesis de alto rendimiento (fotosíntesis C4), similar al maíz o la caña de azúcar, que le permite generar una mayor cantidad de biomasa. Debido a estas peculiaridades fisiológicas, en condiciones óptimas, el jacinto de agua es capaz de duplicar su biomasa entre 512 días, según GIC (2006) se han observado tasas de reproducción en las que partiendo de 100 propágulos nuevos por individuo, la especie es capaz de multiplicar su área inicial por 20 en un plazo de 4 semanas.

Entre los factores antrópicos destacan la falta de medidas adecuadas en la gestión en los medios fluviales y humedales, junto a una carencia de medidas de control en relación con la introducción y cultivo de flora alóctona. Además el cambio climático global, inducido por el hombre, ha favorecido las condiciones adecuadas para la invasión en áreas, que en la primera mitad del siglo $X X$, debido a las bajas temperaturas y el régimen de heladas y nevadas, no eran propicias para su asentamiento.

La convergencia de todos estos factores explica la rápida velocidad de colonización como se evidencia en el Lago Victoria (Tanzania-Kenia-Uganda). Este lago, el de mayor extensión del planeta $\left(69.482 \mathrm{Km}^{2}\right.$ superficie / $3.340 \mathrm{Km}$ perímetro), soporta desde hace décadas una negligente gestión que ha favorecido la introducción de numerosas especies exóticas que han desencadenado graves problemas ambientales y sociales. En 1989 se detectó por vez primera la presencia de Eichhornia crassipes en el Lago Victoria y en 2004 ocupaba una superficie de 17.000 ha (Albright et al. 2004).

Sin embargo, su expansión territorial ha sido más dramática en Asia, América del Sur y en América del Norte. En este último área, el jacinto de agua se encuentra presente en Ontario (Canadá) y en 24 Estados de los EEUU, detectándose invasiones con un gran impacto ecológico al menos en tres de ellos: Carolina del Norte, Carolina del Sur y Miami.

Dado su tamaño y su rápido crecimiento, una vez que un medio acuático es colonizado por el jacinto de agua sus impactos son fácilmente detectables tanto a nivel del funcionamiento ecológico (modificación disponibilidad de nutrientes, interferencia en el intercambio de gases y calor entre las aguas superficiales y profundas, incremento de materia orgánica en los sedimentos superficiales, etc.), como en la modificación de la estructura de los hábitats acuáticos y en su composición florística y faunística. En los ecosistemas acuáticos ubicados en territorios mediterráneos y templados se produce una evidente pérdida de biodiversidad, al desplazar a los elementos nativos, que en muchos casos su persistencia esta vinculadas a condiciones particulares del medio acuático que el desarrollo masivo del jacinto de agua transforma de forma drástica.

Otros efectos de la infestación del Jacinto de Agua se vinculan con su capacidad de servir como hábitat para distintas especies de insectos consideradas como plagas de cultivos, o incluso vectores de enfermedades para el ganado o el hombre. También se ha indicado su interferencia en la navegación, la obstrucción de infraestructuras hidráulicas, y en el desarrollo de actividades de uso recreativo.

En términos generales y en base a las proyecciones y modelos de diversos factores relacionados con el cambio climático, es probable que el jacinto de agua amplíe su distribución global. El Jacinto de agua está presente en la actualidad en diferentes zonas del sur de Europa, pero 
podría fácilmente expandirse al resto de la cuenca mediterránea y más hacia el norte de Europa teniendo en cuenta las previsiones de que aumenten las tasas de calentamiento global (EPPO 2008).

Por todo ellos la IUCN (Unión Internacional para la Conservación de la Naturaleza) considera el Jacinto de Agua como una de las 100 especies invasoras más dañinas del planeta (Lowe et al. 2000).

\section{Distribución y situación en la Península Ibérica}

En Europa el Jacinto de Agua está presente en Portugal, España e Italia. La primera noticia sobre la naturalización en Europa corresponde al año 1940 en Portugal (de Almeida \& Freitas, 2006).Desde esta fecha y hasta la actualidad el
Jacinto de Agua se ha ido extendiendo por el territorio de Portugal continental (Moreira et al. 2002; Martins et al. 2006; Lopes de Almeida, 2006; Monteiro et al. 2010), invadiendo preferentemente ríos y humedales de la zona Centro y Sur, y en menor medida en la Norte (Fig. 3).

Los medios acuáticos más afectados se vinculan con los cursos del Sado, Sorraia y Tejo en la zona Sur y del Mondego, Vouga, Agueda, en la zona Centro, mientras que en la zona Norte su presencia es menos destacada, y solamente se conoce en 3 localidades del río Cávado y 1 en el río Ave, ambas en el distrito del Minho.

En los últimos años se desarrollaron en Portugal numerosas actuaciones para el control y erradicación de la especie, empleando tanto métodos químicos como mecánicos. Pese a ello, el jacinto de agua afecta de forma muy significativa al estado de conservación de numerosos medios acuáticos.

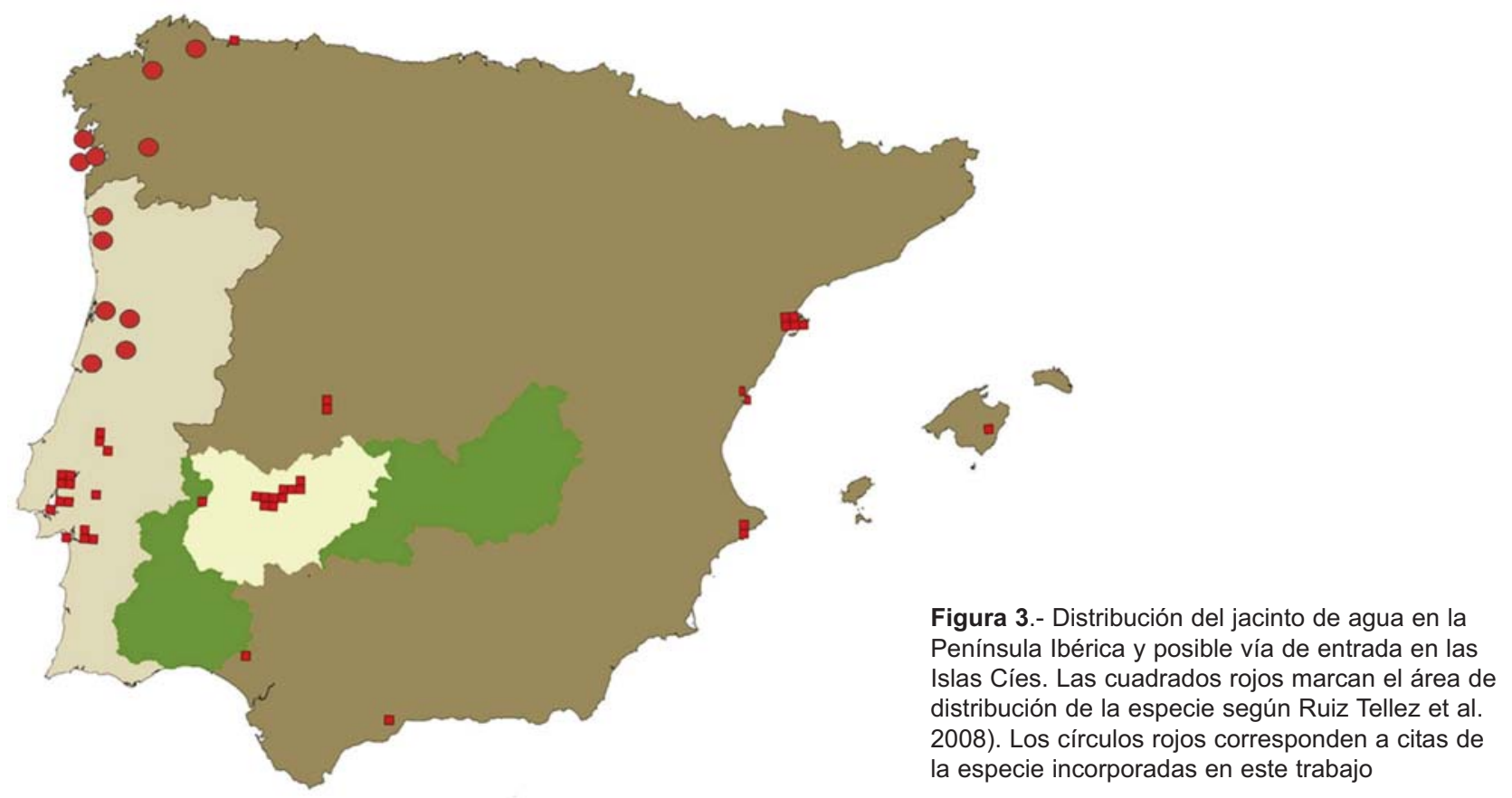

Así a Pateira de Fermentelos, medio lagunar de 525ha situado entre los ayuntamientos de Aveiro, Agueda y Oliveira do Barrio, cerca de la confluencia de los ríos Certima y Agueda, ha sido invadido por numerosas especies exóticas, entre ellas el jacinto de agua (Lopes de Almeida, 2006).Las actuaciones manuales y mecánicas han reducido la intensidad de la invasión, pero pese a ello, la especie mantiene una importante presencia en el humedal.

El humedal de Paul de Boquilobo se sitúa entre la confluencia del río Almonda y el río Tejo. El humedal está catalogado como una Reserva Natural e integrado en la Red Mundial de Reservas de la Biosfera en el año 1981. Las numerosas especies exóticas que en tiempos recientes se han introducido en este humedal han generado importantes desequilibrios ecológicos (Cruz et al. 2008). Entre las especies vegetales, la situación más alarmante es la generada por el desarrollo masivo del jacinto de agua, cuya mitigación obligó a establecer distintas actuaciones de control manual y mecánico. Pese a ello, el grado de infestación sigue siendo muy preocupante.

En el estado español, su introducción parece datarse a finales del siglo XX. En el área mediterránea está presente en distintas localidades (Fig. 3): En 1988 se detecta por primera vez su presencia en un riachuelo próximo a la localidad alicantina de Bolulla, en años posteriores se registrará en Baleares, Badajoz, Cáceres, Castellón y Tarragona (Sanz-Elorza et al. 2004; GEIB, 2006; Ruíz Téllez et al. 2008).

En el año 2001 invadió unas lagunas de agua dulce situadas dentro del Parque Natural del Delta del Ebro (Ullals de l'Arispe), necesitándose para su eliminación un 
programa de actuación aplicado por los técnicos del parque, que consistió en la retirada manual de todas las plantas presentes (Sanz-Elorza et al. 2004). Posteriormente en el 2004 se constata su presencia en Badajoz en la cuenca del río Guadiana donde en pocos años llega a invadir, favorecido por las condiciones climáticas e hidrológicas, amplios tramos del curso fluvial. Las labores de mitigación del Jacinto de Agua en el Guadiana realizadas entre los años 2006-2012 ha supuesto un coste de 21.700.000 euros (Cifuentes, 2012). En el año 2012, los 20 operarios de la Confederación Hidrográfica, retiraron más de 2.000 toneladas, con una media de más de 35 toneladas por día (Fig. 4).
En el área Atlántica peninsular, solo se conocen datos relativos a su presencia en tres provincias: Asturias, Lugo y A Coruña (Fig. 3 y 5). La población de Asturias (González Costales, 2007), fue descubierta en el año 2004, por la Guardería del Medio Natural y corresponde con una única población que había invadido una charca artificial en la localidad costera de Arnao (Castropol), que forma parte de la actual Reserva de la Biosfera del río Eo, Oscos y Terras de Burón. Esta población fue erradicada en una actuación acometida por la Consejería de Medio Ambiente, Ordenación del Territorio e Infraestructuras en el año 2004, aunque en el año 2005 y 2006 todavía pudimos observar varios individuos de escasas dimensiones en sus márgenes.

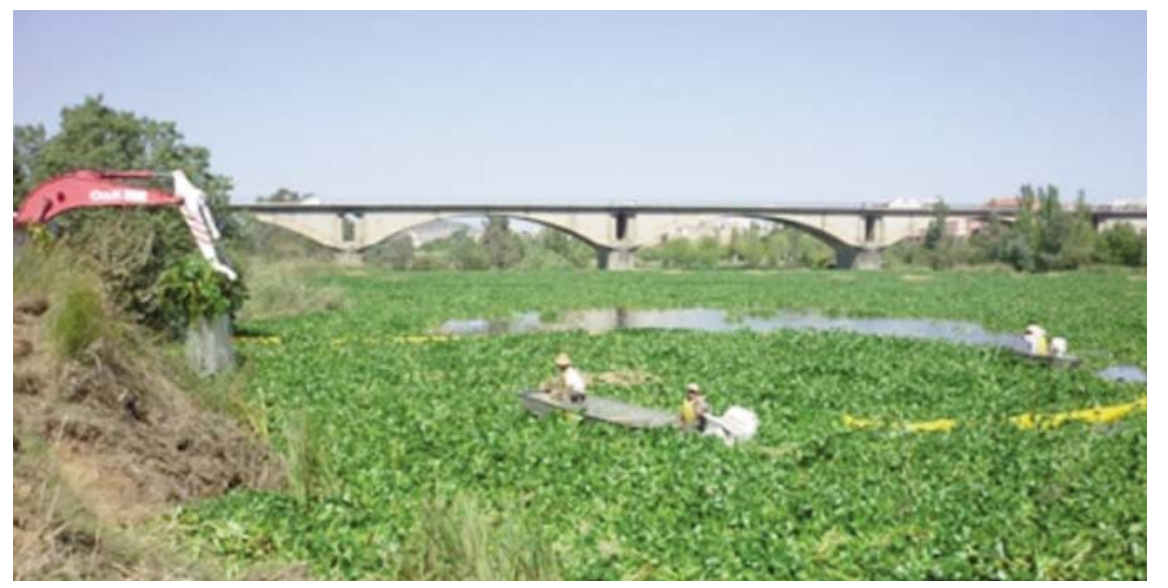

Figura 4.- Eliminación del Jacinto de Agua en el río Guadiana. Fuente: Ministerio de Agricultura, Alimentación y Medio Ambiente
En Galicia su introducción, según hemos podido comprobar se produciría entre los años 1986 y 1988, al ser empleada como elemento ornamental en algunos estanques públicos de As Mariñas Coruñesas. A pesar de la antigüedad de la introducción la especie no ha ocupado otros de medios acuáticos naturales próximos al estanque artificial en el que fue implantada.

En febrero del año 2000, se erradica una pequeña población que ocupaba unos $20 \mathrm{~m}^{2}$ de la laguna de Balea (Cangas, Pontevedra), actuación que fue citada por Pino Pérez et al. (2010). Estos mismos autores, recogen diversos ejemplares varados en la costa del Morrazo, especulando que su procedencia estaría ligada a la existencia de poblaciones en los ayuntamientos de Baiona, Nigrán y Vigo, cuyos restos serían transportados hacia la Península del Morrazo.

Posteriormente hemos podido constatar su presencia en un pequeño estanque artificial en Mondoñedo (Provincia de Lugo) en el año 2007, donde se había introducido junto con otras especies ornamentales y algunos individuos jóvenes de carpa. Informado el Servicio Provincial de Conservación de la Naturaleza, este procedió con el asentimiento del propietario, a la eliminación de los elementos exóticos.

En años posteriores se efectuaron medidas de erradicación, por la presencia de Eichhornia crassipes, aunque siempre en número muy reducido, en las lagunas de Bodeira ( $O$ Grove, Pontevedra) y en el tramo medio y bajo del río Avia cerca del embalse de Albarellos (ayuntamientos de Leiro, Beade y Ribadavia todos ellos de la provincia de Ourense).

El Real Decreto 630/2013, de 14 de noviembre, por el que se regula el Catálogo español de especies exóticas invasoras, incluye en su anexo al jacinto de agua (Eichhornia crassipes):

La inclusión de una especie en este catálogo, de acuerdo al artículo 52.2 de la Ley 42/2007, de 13 de diciembre, conlleva la prohibición de su introducción en el medio natural, en todo el territorio nacional y en las aguas marinas sometidas a soberanía o jurisdicción española.

La inclusión de una especie en el Catálogo Español de Especies Exóticas Invasoras conlleva, de acuerdo al artículo 61.3 de la Ley 42/2007, de 13 de diciembre, la prohibición genérica de su posesión, transporte, tráfico y comercio de ejemplares vivos o muertos, de sus restos o propágulos, incluyendo el comercio exterior. Esta prohibición podrá quedar sin efecto, previa autorización administrativa, cuando sea necesario por razones de investigación, salud o seguridad de las personas.

\section{Resultados y discusión}

La llegada de arribazones es un hecho frecuente en las playas del archipiélago de Cíes. En la mayoría de los casos se corresponden con fragmentos de algas marinas o incluso 


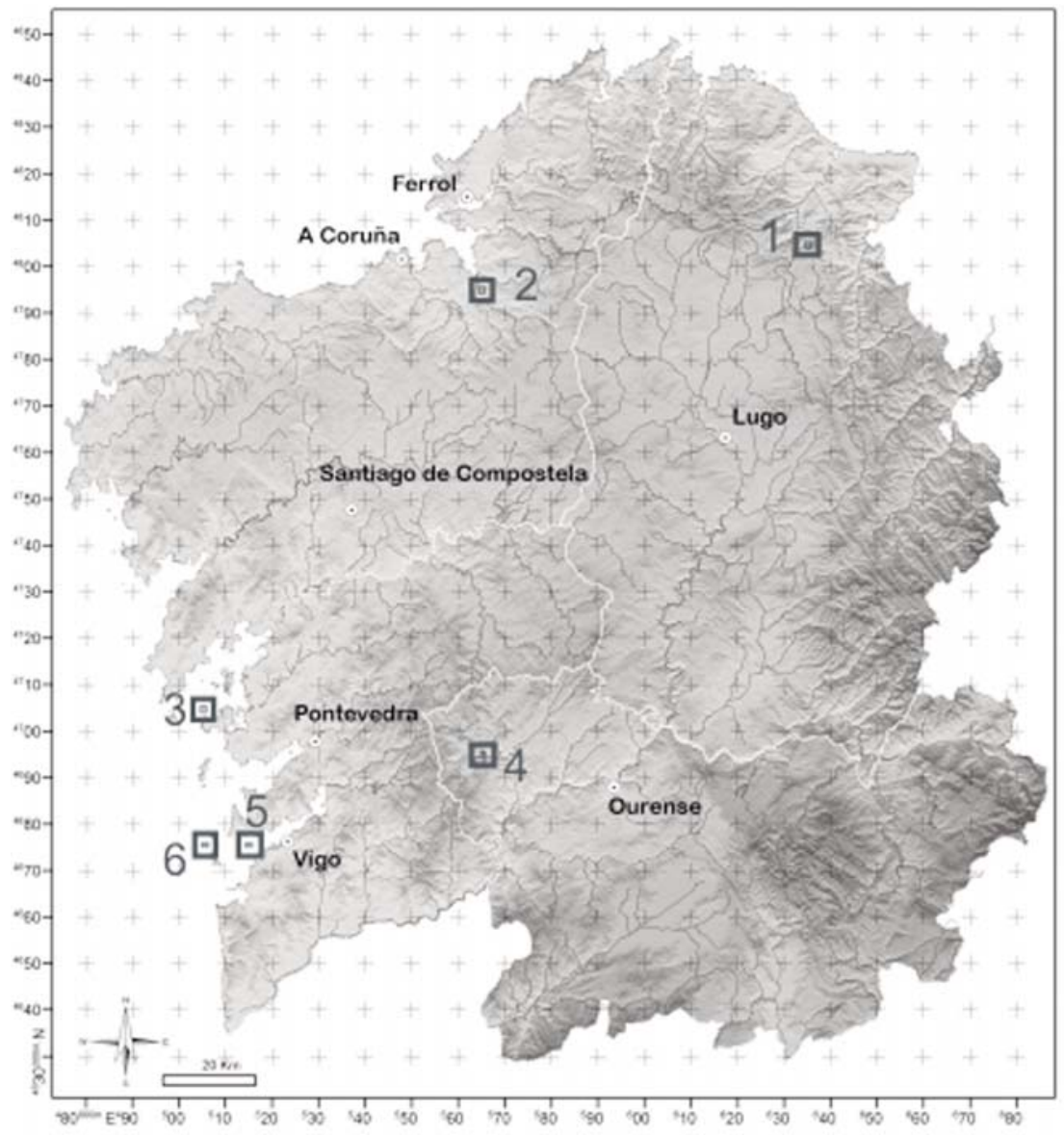

Figura 5.- Citas del Jacinto de Agua (Eichhornia crassipes) en Galicia. 1Mondoñedo; 2- As Mariñas; 3- O Grove, 4- Río Avia; 5- Cangas; 6Illas Cíes de restos (raíces, vástagos, hojas) de plantas, frutos y semillas (bellotas, piñones), procedente de la vegetación que crece en las áreas continentales próximas, encontrándose macro-restos de elementos tanto característicos de medios de cultivo, como de ecosistemas de marisma, dunas o de medios continentales.

En noviembre del 2011 la guardería del Parque Nacional encuentra en la playa de Rodas en el archipiélago de Cíes, (UTM 29T 508.052,89/ 4.674.729,88) una gran cantidad de restos deteriorados de una planta herbácea de grandes dimensiones. La presencia de peciolos hinchados indicaban que los restos arribados podrían pertenecer a una especie de la familia Pontederiaceae, y probablemente al jacinto de agua o camalote (Eichhornia crassipes).

Tras el paso del temporal que barrió el NW Ibérico a mediados del octubre del 2012, el día 18 del mismo mes, el personal del Parque Nacional observó que la playa de Rodas (UTM 29T 508.052,89/ 4.674.729,88) se había cubierto por numerosas plantas de gran tamaño (Figs. 6 a $9)$. En estos ejemplares son visibles los elementos vegetativos característicos (raíces, hojas flotantes con hinchazones en el peciolo, hojas sumergidas) del jacinto de agua o camalote (Eichhornia crassipes). En los ejemplares observados no se aprecian estructuras reproductoras, ni tampoco resto de pedúnculo de la espiga.

En los limbos foliares y también en los pedúnculos se observa una elevada deshidratación, fruto de un contacto prolongado en el agua marina, así como lesiones y cortes a nivel de los tejidos superficiales que probablemente se habrían producido por acción del oleaje (Figs. 6 a 9). El estado de los restos vegetativos no resulta el más adecuado para el mantenimiento de su capacidad regenerativa, pero de cualquier modo no se puede asumir que puedan permanecer yemas o resto de tejidos que puedan desarrollar nuevas estructuras vegetativas.

Por otra parte, las zonas de llegada, sistemas de playa, resultan totalmente inadecuados para el arraigo de estos especímenes. El peligro de los mismos es que desde las playas puedan ser transportados por el viento, o por la acción de animales o del hombre, y ser depositados en medios más adecuados para su mantenimiento.

La posibilidad de invasión en estos medios estaría limitada por la ausencia de restos con semillas viables. En el caso de que esas lleguen a estar presentes en el arribazón, el riesgo de invasión sería muy elevado, ya que los datos científicos corroboran la gran resistencia de estas estructuras y su elevada capacidad germinativa, que llega a mantenerse hasta durante 20 años.

Una vez confirmada la identificación de los ejemplares y evaluado el riesgo, el personal del Parque Nacional procedió a la retirada y destrucción de los restos. 


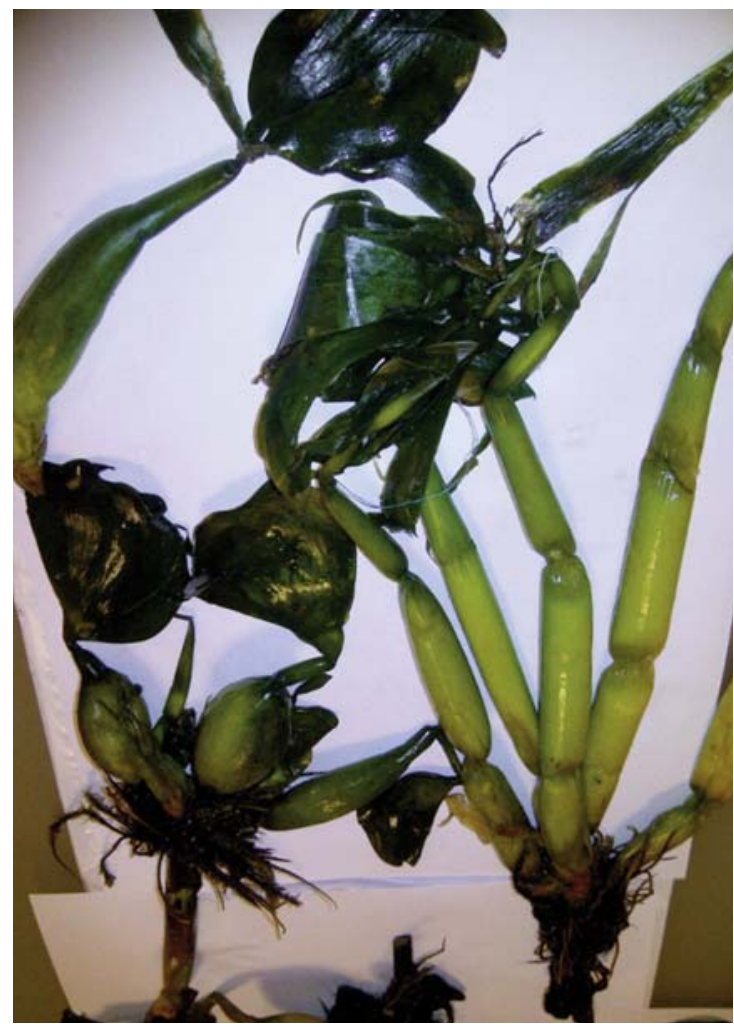

Figura 6.- Fotografía de Jacinto de Agua (Eichhornia crassipes) recuperados en la playa de As Rodas (Islas Cíes), dentro del Parque Nacional Marítimo - Terrestres das Illas Atlánticas de Galicia. Vista general de dos individuos

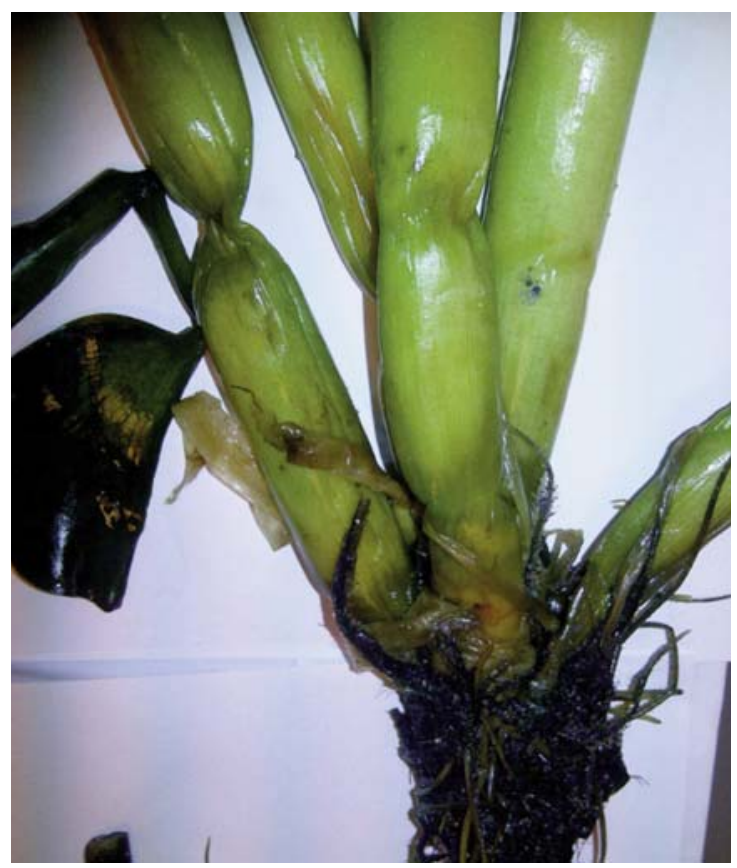

Figura 8.- Fotografía de Jacinto de Agua (Eichhornia crassipes) recuperados en la playa de As Rodas (Islas Cíes), dentro del Parque Nacional Marítimo - Terrestres das Illas Atlánticas de Galicia. Detalle de un individuo con hojas sumergidas carentes de hinchazones en los peciolos

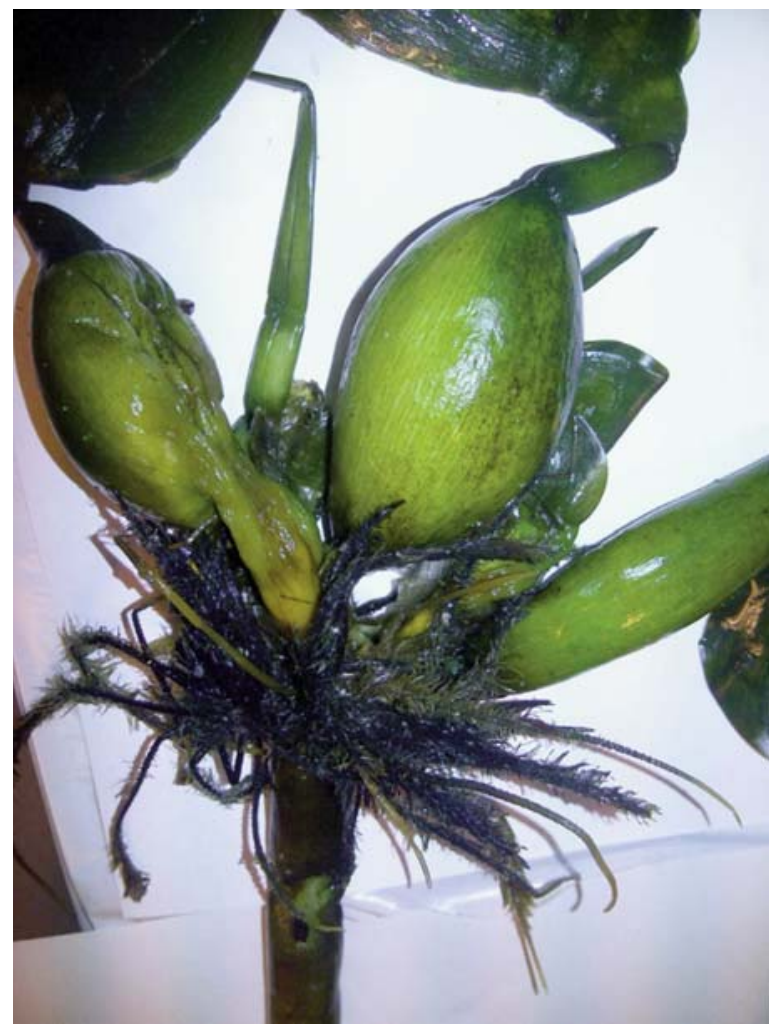

Figura 7.- Fotografía de Jacinto de Agua (Eichhornia crassipes) recuperados en la playa de As Rodas (Islas Cíes), dentro del Parque Nacional Marítimo - Terrestres das Illas Atlánticas de Galicia. Detalle de hojas flotantes, con hinchamientos en el peciolo

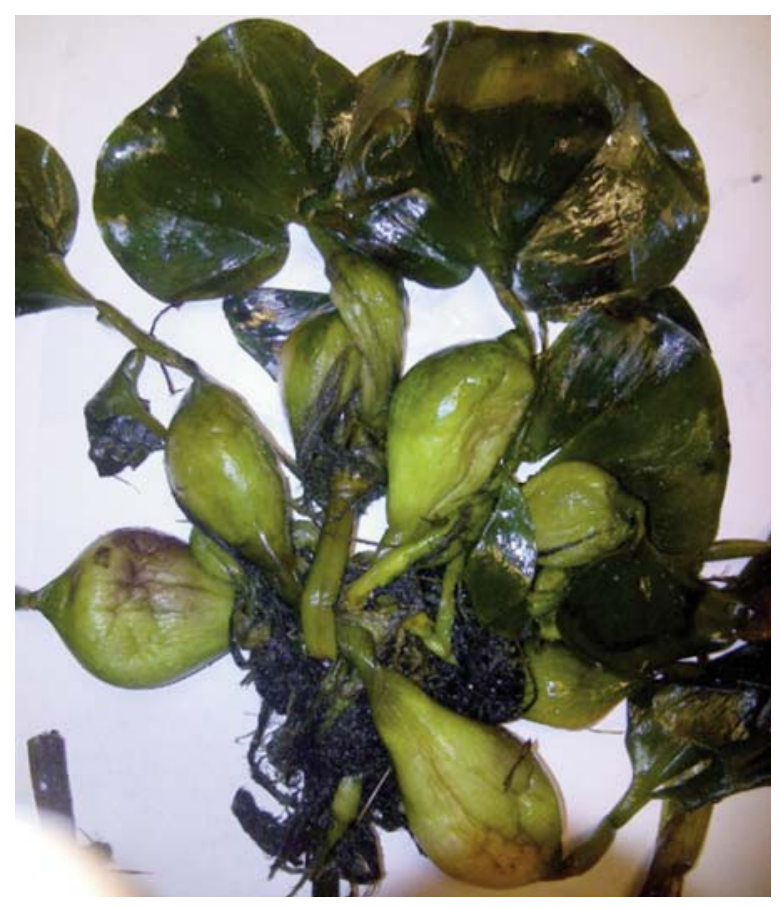

Figura 9.- Fotografía de Jacinto de Agua (Eichhornia crassipes) recuperados en la playa de As Rodas (Islas Cíes), dentro del Parque Nacional Marítimo - Terrestres das Illas Atlánticas de Galicia. Vista general de un individuo con hojas flotantes 
En cuanto al origen de estos arribazones se descarta una fuente local y también una fuente próxima en la costa gallega. El número de plantas llegadas a la playa de Rodas, vincula su procedencia con un área en la que la población de jacinto de agua ha de ser muy importante. En la provincia de Pontevedra, no se conoce ninguna localidad que pueda aportar tal cantidad de planta de jacinto de agua en un único arribazón. La única localidad conocida actualmente en territorio gallego la conforman un número reducido de individuos, localizados en el norte de la provincia de Lugo, que aun en caso de ser transportados por las corrientes marinas, serían enviados hacia localidades más septentrionales y no meridionales.

Descartando una procedencia gallega para estos arribazones, su origen debe vincularse con las abundantes poblaciones existentes en Portugal, bien en relación con las localidades del Norte o preferentemente, dado el tamaño de estas, con poblaciones existentes en la zona Centro de Portugal.

\section{Bibliografía}

Albright, T.P., Moorhouse, T.G. \& McNabb T.J. (2004). The rise and fall of water hyacinth in Lake Victoria and the Kagera River Basin, 1989-2001. Journal of Aquatic Plant Management. 42: 73-84.

Amaral Franco,J. \& Rocha Alfonso, M.L. (1994). Nova Flora de Portugal, 3 (1). Escolar Editora, Lisboa.

Cifuentes, N. (2012). El Jacinto de agua (Eichhornia crassipes) en la cuenca del Guadiana, experiencias de manejo. Jornadas sobre especies invasoras de ríos y zonas húmedas.

Valencia.

http://www.cma.gva.es/web/indice.aspx?nodo=78328\&idio ma $=$ C.

Cruz, J.M.; Segurado, P.; Sousa, M. \& Rebelo, R. (2008). Collapse of the amphibian community of the Paul do Boquilobo Natural Reserve (central Portugal) after the arrival of the exotic American crayfish Procambarus clarkia. The Herpetological Journal. 18, 4: 197-204.

de Almeida, J.D. \& Freitas, H. (2006). Exotic naturalized flora of continental Portugal - A reassessment. Botanica Complutensis. 30: 117-130.

Dias, J.M.A., Gonzales, R., García, C. \& Diaz-del-Río, V. (2002). Sediment distribution pattern on the Galicia-Minho continental shelf. Progress In Oceanography. 52: 215-231.

Ding, J., Wang, R., Fu, W. \& Zhang, G. (2001).Water hyacinth in China: its distribution, problems and control status. In: Julien, M.H., Hill, M.P., Center, T.D. \& Ding, J. (Ed.) Proceedings of the 2nd Meeting of the Global Working Group for the Biological and Integrated Control of Waterhyacinth. Beijing, China. 29-32.
EPPO (2008). Pest Risk Analysis for Eichhornia crassipes. 57 p. http://www. eppo.org/QUARANTINE/Pest_Risk_Analysis/PRAdocs_pla

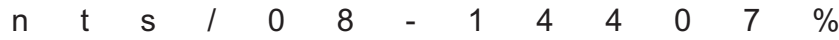
20PRA\%20record\%20Eichhornia\%20crassipes $\% 20$ EICCR. pdf.

GEIB: Grupo Especialista en Invasiones Biológicas (2006). TOP 20: Las 20 especies exóticas invasoras más dañinas presentes en España. GEIB, Serie Técnica N2. León.

GIC: Grupo de Investigación en Biología de la Conservación de la Universidad de Extremadura (2006). Informe sobre Distribución y Biología Reproductora del jacinto de agua en el Guadiana, 12 vols. Confederación Hidrógráfica del Guadiana. Ministerio de Medio Ambiente. Badajoz, España.

Gómez-Orellana, L., Ramil-Rego, P. \& Muñoz Sobrino, C. (2013). The response of vegetation at the end of the last glacial period (MIS 3 and MIS 2) in littoral Areas of NW Iberia. Boreas. 44: 729-744.

González Costales, J.A. (2007). Plantas alóctonas invasoras en el Principado de Asturias. Consejería de Medio Ambiente, Ordenación del Territorio e Infraestructuras y Obra Social "la Caixa”. Asturias.

Gopal, B. (1987) Water Hyacinth. Elsevier. Amsterdam.

Lopes de Almeida, A.S. (2006).Avaliação da qualidade da água na Pateira de Fermentelos - caso de estudo na Área de Projecto. Universidade de Aveiro. Departamento de Biologia. Dissertaçâo Grau de Mestre em Ciemcias das Zonas Costeiras. Aveiro.

Lowe S.J., Browne, M. \& Boudjelas, S. (2000). 100 of the World's Worst Invasive Alien Species. IUCN/SSC Invasive Species Specialist Group (ISSG). Auckland, New Zealand.

Martins, A.M., do S. Raposo, J.M., Pimentel, M.H., Silveira, S.M., de Sousa, A.C. \& Raimundo, S.M. (2006). Bases para um plano de requalificaçâo das Lagoas do litoral da Regiâo Centro. Comissâo de Coordenaçâo e Desenvolvimento Regional do Centro. Divisâo do Litoral e da Conservaçâo da Natureza. Coimbra.

Monteiro, A., Moreira, I., Serrasqueiro, P.M. \& Santos, A.C. (2010). Jacinto-aquatico (Eichornia crassipes) em Portugal. Bioecologia e gestâo.

Moreira, I., Ferreira, M.T., Cortes R., Pinto, P. \& Almeida, P.R. (2002). Ecossistemas Aquáticos e Ribeirinhos. Ecologia, Gestão e Conservação. Instituto da Água, MCOTA. Lisboa.

Owens, C.S. \& Madsen J.D. (1995). Low temperature limits of water hyacinth. Journal of Aquatic Plant Management. 33: 63-68.

Pino Pérez, J.J., Rial Pousa, D., Silva-Pando, F.J., Camaño Portela, J.L. \& Pino Pérez, R. (2010). Contribución a la flora alóctona de Galicia. Boletín Auriense. XXXVIII-XXXIX: 385402. 
Ruiz Tellez, T., de Rodrigo López, E.M., Lorenzo Granado, E.;, Albano Pérez, E., Morán López, R. \& Sánchez Gúzman, J.M. (2008). The water hyacinth, Eichhornia crassipes: an invasive plant in the Guadiana River Basin (Spain). Aquatic Invasions. 3, 1: 42-53.

Sanz Elorza M., Dana Sánchez E.D. \& Sobrino Vesperinas E. (2004). Atlas de las plantas alóctonas invasoras en España. Dirección General para la Biodiversidad. Madrid.

Sato, H. (1988). The growth analysis of water hyacinth, Eicchornia crassipes (Mart) Solms, in different water temperature conditions. Ecological Research. 3: 131-144. von Martius, C.F.P. (1823-1832). Plantarum Brasiliensium Nova genera et species novae, vel minos cognitae (1823-1832). Tipographia Aloisii Pezzati. Florentiae.

Wilson, J.R., Holst, N. \& Rees, M. (2005). Determinants and patterns of population growth in water hyacinth. Aquatic Botany. 81: 51-67. 